\title{
Fitorremediação de Solo Contaminado por Metais Pesados
}

\author{
Soil Method Contaminated Soil Phytoremediation
}

\author{
Marissol Leite ${ }^{1}$ \\ Elenara Pereira Ventura Guajajara ${ }^{1}$ \\ Sanderson Silva ${ }^{1}$ \\ Wesley Lima da Silva ${ }^{1}$ \\ Sidnei Cerqueira dos Santos ${ }^{1}$ \\ ${ }^{1}$ Universidade Federal do Sul e Sudeste do Pará, Marabá, PA, Brasil
}

\begin{abstract}
Resumo
A fitorremediação é uma técnica que utiliza plantas para remediar áreas contaminadas com metais pesados. Essa técnica possui grande vantagem devido à alta diversidade de vegetais que podem ser encontrados na natureza. Este trabalho teve como objetivo fazer o levantamento tecnológico dos métodos de fitorremediação usados na recuperação de ambientes impactados por metais pesados. A pesquisa foi realizada nos bancos de dados de patentes do Escritório Europeu de Patentes (Espacenet ${ }^{\circledR}$ ) e do Instituto Nacional de Propriedade Industrial (INPI), utilizando as palavras-chave: remediação, planta, solo, metal pesado, recuperação e fitorremediação, e os códigos B09C1/00 e A01H5/00 da Classificação Internacional de Patentes. A China foi o destaque nesta pesquisa, apresentando os principais inventores e universidades detentoras de patentes, tendo o auge de depósito no ano de 2009. A técnica de fitorremediação mais utilizada foi a fitoextração. As tecnologias fitorremediadoras se mostram bastante promissoras para atender o mercado que é cada vez mais exigente e preocupado com os impactos ambientais.
\end{abstract}

Palavras-chave: Prospecção tecnológica. Biorremediação. Fitoextração. Mineração.

\begin{abstract}
Phytoremediation is a technique that uses plants to remedy areas contaminated with heavy metals. This technique has great advantage due to the high diversity of vegetables that can be found in nature. This work aimed to make a technological survey of the phytoremediation methods used in the recovery of environments impacted by heavy metals. The research was carried out in the patent databases of the European Patent Office (Espacenet $\left.{ }^{\circledR}\right)$ and the National Institute of Industrial Property (INPI), using the keywords: remediation, plant, soil, heavy metal, recovery and phytoremediation, and codes the $\mathrm{B} 09 \mathrm{C} 1 / 00$ and $\mathrm{A} 01 \mathrm{H} 5 / 00$ of the International Patent Classification. China was the highlight in this research, presenting the main inventors and universities holding patents, having the peak of deposit in the year 2009. The phytoremediation technique that is being more used is phytoextraction. Phytoremediative technologies are proving to be very promising for a market that is increasingly demanding and concerned about environmental impacts.
\end{abstract}

Keywords: Technological prospecting. Bioremediation. Phytoextraction. Mining.

Área tecnológica: Interdisciplinar. Biotecnologia. 


\section{Introdução}

O solo tem grande importância para a atividade humana, sendo fonte de produção de alimento, combustível, material de construção, o que torna sua preservação imprescindível para a sobrevivência dos seres vivos (RIBEIRO, 2013). No entanto, com o advento da revolução industrial e o desenvolvimento tecnológico, até os dias atuais, a poluição do solo vem aumentando consideravelmente, comprometendo a qualidade de vida dos seres vivos e dos variados ecossistemas da Terra. As principais fontes dessa poluição são as atividades agrícolas e minerais que ocorrem de maneira desenfreada, gerando sérios danos ao meio ambiente (SOBRINHO et al., 2009).

Muitas indústrias mineradoras ao realizarem suas atividades não dão a devida importância para as normas de biossegurança, e exploram o solo de maneira inadequada, ocasionando grande impacto na superfície terrestre, principalmente pelos rejeitos constituídos por metais pesados, como $\mathrm{Hg}, \mathrm{Cd}, \mathrm{Pb}$, que são altamente tóxicos (CAMPOS et al., 2003).

Outra forma de contaminação do solo está relacionada com a atividade agrícola, onde é usada uma grande quantidade de fertilizantes em diferentes tipos de plantações, acarretando numa hiperacumulação de substâncias tóxicas no solo, causando poluições muitas vezes irreversíveis (RIBEIRO, 2013).

A multifuncionalidade do solo acaba por ser um paradoxo, pois a sua importância para um vasto leque de atividades humanas torna-o mais vulnerável aos danos e às exaustões provocadas por estas (RIBEIRO, 2013). Infelizmente, em várias partes do mundo, inclusive no Brasil, existem diversas áreas com solos contaminados (SILVA, 2012). Nesse sentido, muitos pesquisadores vêm desenvolvendo técnicas de biorremediação do solo, ou seja, métodos para convertê-lo em seu estado primário/natural ou menos tóxicos, como a fitorremediação.

A fitorremediação é uma tecnologia que utilizar plantas para remover, conter, transferir, estabilizar e/ou degradar compostos orgânicos e metais tóxicos (RASKIN; ENSLEY, 2000).

A busca em base de dados de patentes é uma ferramenta importante para identificação do grau de desenvolvimento dos processos tecnológicos, que são geralmente protegidos na forma de patente de invenção e modelo de utilidade (INPI, 2017). Dessa forma, o objetivo deste trabalho foi avaliar o desenvolvimento tecnológico dos processos de fitorremediação de solo contaminado por metais pesados, com o intuito de conhecer quais as inovações tecnológicas vêm sendo desenvolvidas sobre a temática.

\section{Metodologia}

\subsection{Buscas em Bases de Patentes}

As buscas de patentes foram realizadas em duas plataformas online de pesquisa de patentes: Instituto Nacional de Propriedade Industrial (INPI) e Escritório Europeu de Patentes (Espacenet $\left.{ }^{\circledR}\right)$, ambas possuem acesso gratuito. 


\subsection{Seleção de Palavras-chave e Códigos}

A pesquisa básica foi realizada usando as palavras-chave: remediação (remetiation). A pesquisa avançada foi realizada usando as palavras-chave: remediação (remediation), planta (plant), solo (soil), metal pesado (heavy metal), recuperação (recovery) e fitorremediação (phytoremediation), selecionadas com base no escopo do trabalho. As palavras-chave foram inseridas no campo Título e Resumo de ambas as bases de dados de patentes. Os códigos internacionais de patentes (IPC): B09C1/00 (Recuperação de solo contaminado/por processos microbiológicos ou mediante o uso de enzimas) e A01H5/00 (Plantas floríferas, i.e. angiospermas) foram usados também na pesquisa.

\subsection{Escopo}

O operador booleano (AND) e o operador de truncagem (*), que permite encontrar possíveis derivações das palavras utilizadas, foram usados na pesquisa avançada para combinar palavras-chave no título e no resumo (Tabela 1). Os códigos selecionados também foram combinados na pesquisa avançada com as palavras-chave (Tabela 1). As patentes selecionadas foram processadas e apresentadas por meio de gráficos, em termos do cenário tecnológico e desenvolvimento da tecnologia.

Tabela 1 - Escopo de busca

\begin{tabular}{|c|c|c|c|c|c|c|c|c|c|}
\hline \multicolumn{6}{|c|}{ Palavras-Chave } & \multicolumn{2}{|c|}{ Códigos } & \multicolumn{2}{|c|}{ EscritóRIOS } \\
\hline Remedi* & Plant & soil & $\begin{array}{l}\text { Heavy } \\
\text { Met* }\end{array}$ & Recov* & Phytoremed* & B09C $1 / 00$ & $\mathrm{~A} 01 \mathrm{H} 5 / 00$ & INPI & ESPACENET \\
\hline$X$ & & & & & & & & 31 & 7.320 \\
\hline $\mathrm{X}$ & $\mathrm{X}$ & & & & & & & 0 & 577 \\
\hline $\mathrm{X}$ & & $\mathrm{X}$ & & & & & & 8 & 2.852 \\
\hline \multirow[t]{10}{*}{$\mathrm{X}$} & & & $\mathrm{X}$ & & & & & 1 & 332 \\
\hline & & & & & $\mathrm{X}$ & & & 1 & 327 \\
\hline & $\mathrm{X}$ & & & $\mathrm{X}$ & & & & 9 & $>10.000$ \\
\hline & $\mathrm{X}$ & & $\mathrm{X}$ & & & & & 1 & 1.123 \\
\hline & & $\mathrm{X}$ & $\mathrm{X}$ & & & & & 0 & 2.218 \\
\hline & & $\mathrm{X}$ & & $\mathrm{X}$ & & & & 5 & 2.268 \\
\hline & & $\mathrm{X}$ & & & $\mathrm{X}$ & & & 0 & 212 \\
\hline & & & $\mathrm{X}$ & $\mathrm{X}$ & & & & 2 & 908 \\
\hline & & & $\mathrm{X}$ & & $\mathrm{X}$ & & & 0 & 53 \\
\hline & & & & & & $\mathrm{X}$ & & 35 & 8.144 \\
\hline \multirow[t]{8}{*}{$\mathrm{X}$} & & & & & & $\mathrm{X}$ & & 6 & 1.207 \\
\hline & & $\mathrm{X}$ & & & & $\mathrm{X}$ & & 6 & 4.288 \\
\hline & & & & $\mathrm{X}$ & & $\mathrm{X}$ & & 1 & 281 \\
\hline & & & $\mathrm{X}$ & & & $\mathrm{X}$ & & 0 & 842 \\
\hline & $\mathrm{X}$ & & & & & $\mathrm{X}$ & & 0 & 657 \\
\hline & & & & & $\mathrm{X}$ & $\mathrm{X}$ & & 0 & 120 \\
\hline & & & & $\mathrm{X}$ & & & $\mathrm{X}$ & 0 & 127 \\
\hline & $\mathrm{X}$ & & $X$ & & & $X$ & & 0 & 215 \\
\hline
\end{tabular}

Fonte: Elaborada pelos autores deste artigo 


\section{Resultados e Discussão}

As patentes analisadas são provenientes da combinação entre as palavras-chave "plant and heavy metal", com o código B09C1/00, na busca realizada no Espacenet ${ }^{\circledR}$. Utilizando essa combinação obteve-se 215 patentes, sendo selecionadas manualmente 99 delas para fins de análise, depois da triagem de documentos repetidos e relacionados efetivamente com o tema da pesquisa. As patentes encontradas no INPI não foram utilizadas nesta pesquisa, pois os documentos encontrados a partir das combinações de palavras-chave e códigos tinham pouca relação com o tema proposto neste trabalho ou estava disponível na busca realizada no Espacenet ${ }^{\circledR}$.

Figura 1 - Patentes depositadas por país

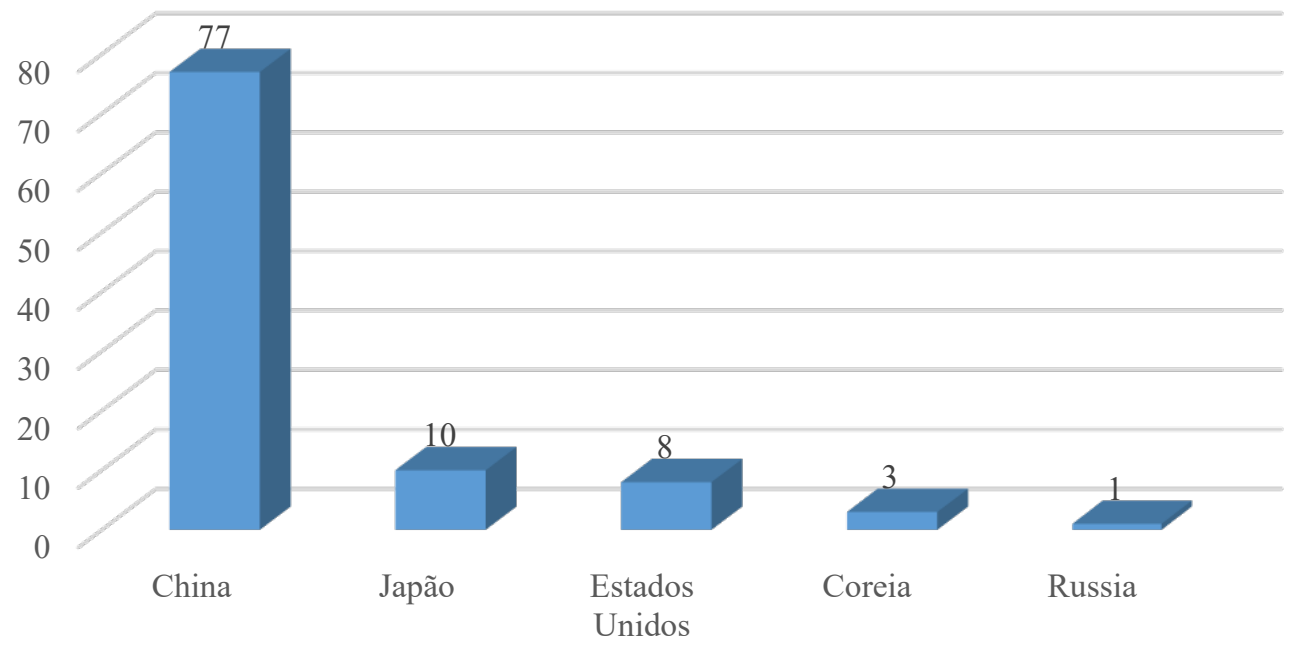

Fonte: Elaborada pelos autores deste artigo (2018)

A Figura 1 apresenta o número de depósitos de patentes por países, com a China liderando o número de depósitos quando comparado com os demais países. A China é uma das potências mundiais, portanto, é um dos países que mais investe em novas tecnologias, além disso, é muito rica em diversidade biológica, chegando em torno de 31 mil espécies de plantas nativas (ZUCOLOTO, 2013), o que propicia o estudo em novos ramos de pesquisa que evidenciem o equilíbrio da natureza e o bem-estar da população.

Além das vantagens naturais, a China apresenta razão adicional para investir na biotecnologia, pois é um dos países mais populosos do mundo. As preocupações relacionadas à alimentação e saúde tornam-se prioridade nas pautas de políticas públicas da nação (ZUCOLOTO, 2013). A biotecnologia é uma alternativa para solucionar problemas urgentes, como aumento da população, fornecimento de alimento, cuidados médicos e proteção ambiental (MAYERHOFF, 2007).

O governo da China recentemente reconheceu que 16,1\% do território chinês está contaminado, incluindo metais pesados, como cádmio, mercúrio, arsénico e chumbo. Isso explica por que a China tem estado na frente dos outros países quando o assunto diz respeito à recuperação de solo contaminado por metais pesados (PUBLICO, 2014). 
Figura 2 - Patentes depositadas por ano

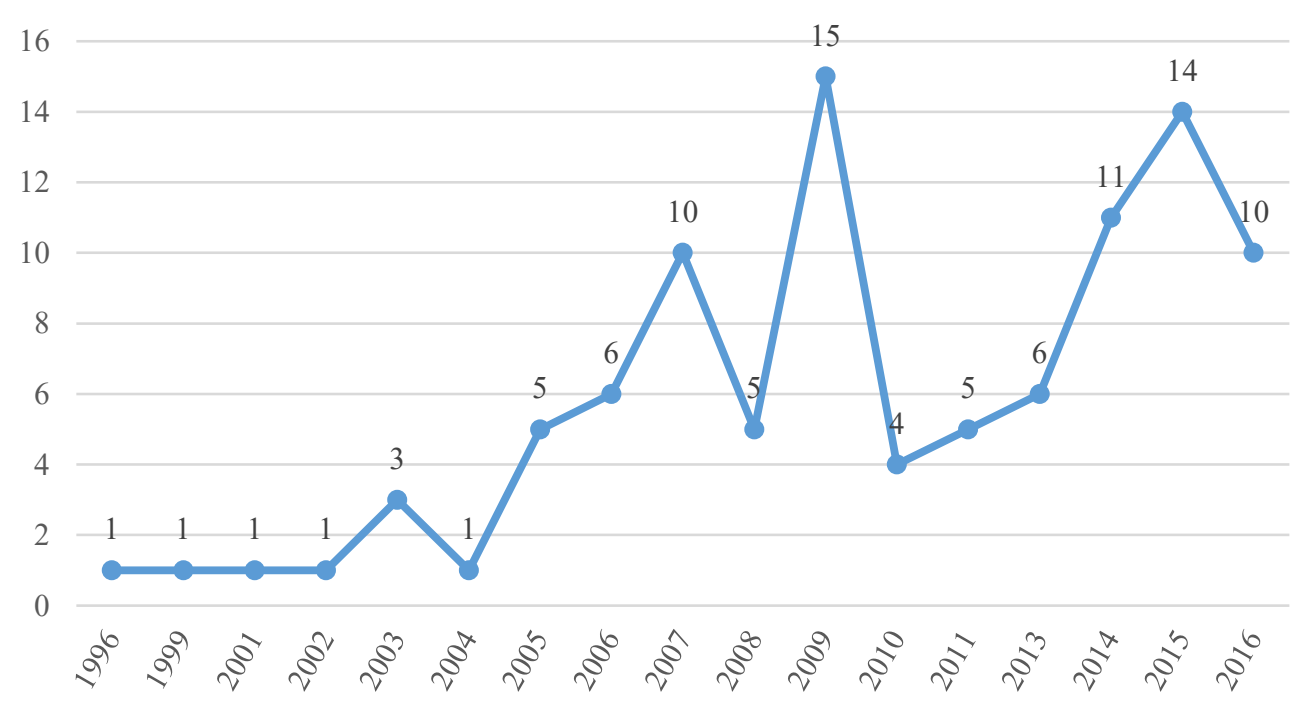

Fonte: Elaborada pelos autores deste artigo (2018)

Como mostra a Figura 3, a maioria dos pedidos de patentes é proveniente de universidades, perfazendo mais da metade das patentes depositadas, seguido de empresas privadas, institutos de pesquisa, que também concentram seus estudos nessa área, e, por fim, as pessoas físicas, que apresentaram o menor valor das patentes depositadas.

Figura 3 - Patentes por pessoas jurídica e física

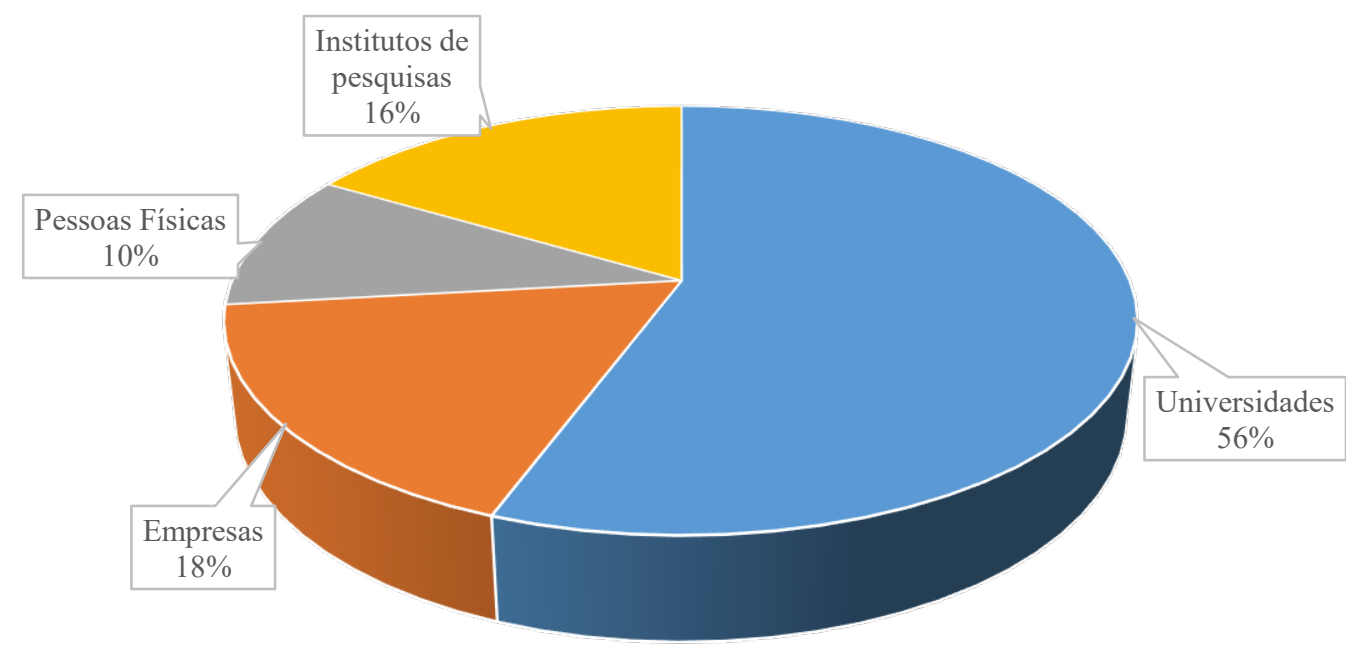

Fonte: Elaborada pelos autores deste artigo (2018)

A Figura 4 mostra os principais inventores, sendo todos de nacionalidade chinesa. Em destaque Shirong Zhang, que possui aproximadamente três vezes mais patentes em comparação com os demais inventores, sendo em sua maioria depositadas pela Universidade Sichuan Agricultural, que possui o maior número de patentes depositadas. 
Figura 4 - Patentes por inventor

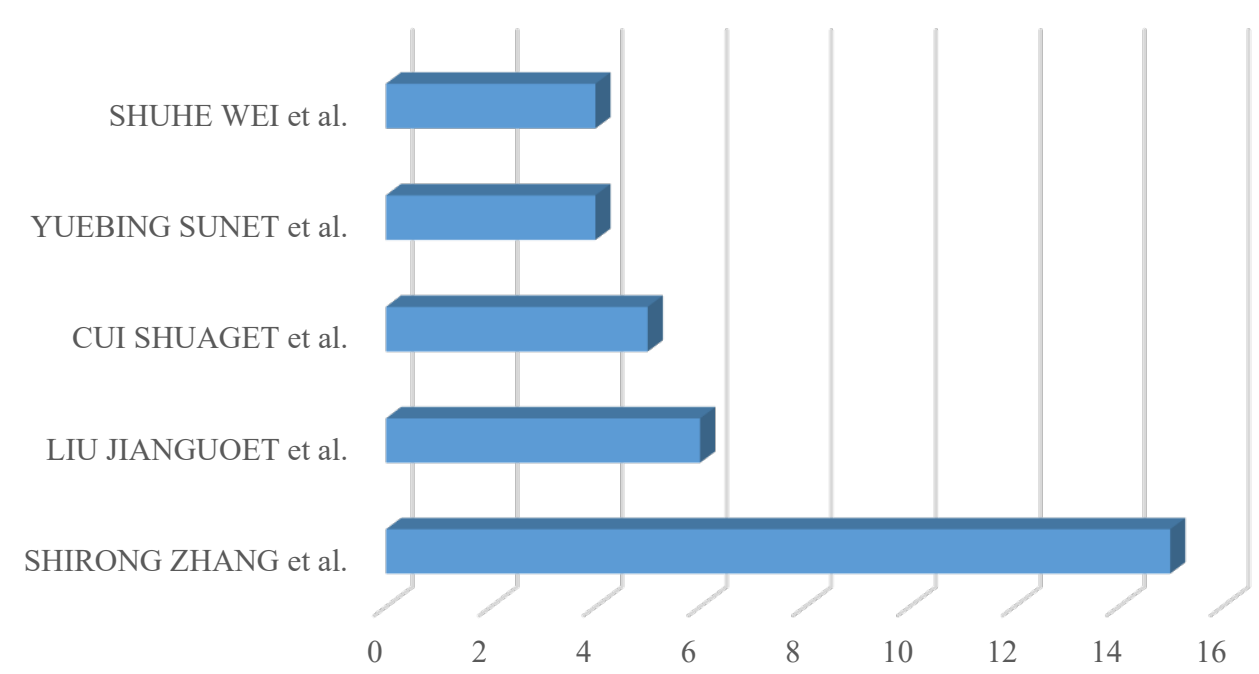

Fonte: Elaborada pelos autores deste artigo (2018)

Na Figura 5 são apresentadas as principais pessoas jurídicas depositantes de patentes, sendo as três primeiras correspondentes a universidades chinesas, seguido de dois institutos de pesquisas. Observando-se que as instituições de origem japonesa têm tido um bom desempenho em suas pesquisas e desenvolvimento tecnológico, o que pode estar relacionado com as condições favoráveis de infraestrutura e de recursos financeiro e humano.

Figura 5 - Patentes por depositante

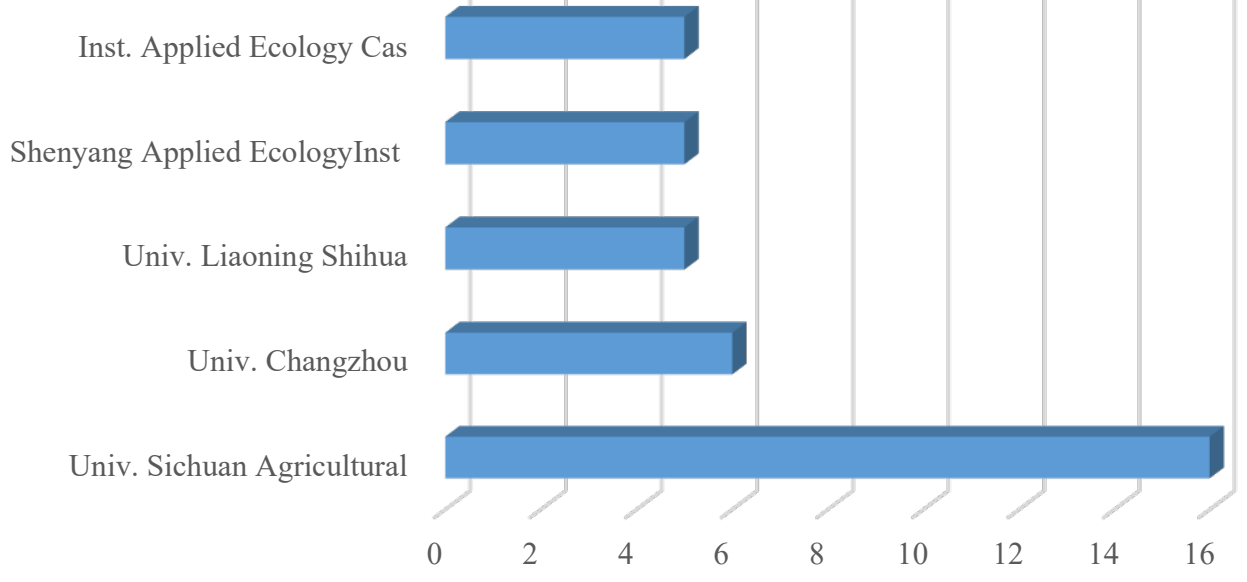

Fonte: Elaborada pelos autores deste artigo (2018)

Os processos de fitorremediação podem ser classificados em fitoestabilização, fitovolatização, fitodegradação, fitoestimulação e fitoextração. A fitoestabilização consiste em limitar a mobilidade do metal pesado no solo, incorporando-o na raiz da planta. Na fitovolatização a planta absorve o metal pesado, converte em um composto menos tóxico e libera para atmosfera. O processo de fitodegradação consiste em degradar compostos tóxicos, utilizando enzimas específicas, no qual tais compostos serão utilizados pela planta como nutriente. Na fitoestimulação a planta utiliza de 
suas raízes em crescimento, com o intuito de proliferar micro-organismos capazes de degradar contaminantes. Por fim, na fitoextração, o vegetal absorve o metal pesado do solo por meio das raízes e o acumula preferencialmente nas partes aéreas (caule e folhas) (NASCIMENTO; XING, 2006; SILVA, 2012). Nesta pesquisa, a fitoextração foi o principal processo fitorremediador protegido por patente, seguida por fitoestabilização, fitovolatização e fitodegradação (Figura 6). Das 99 patentes analisadas, não foi possível identificar o tipo de processo empregado em 10 documentos, pois não havia a caracterização da técnica no corpo do resumo.

Figura 6 - Processos de fitorremediação protegidos por patentes

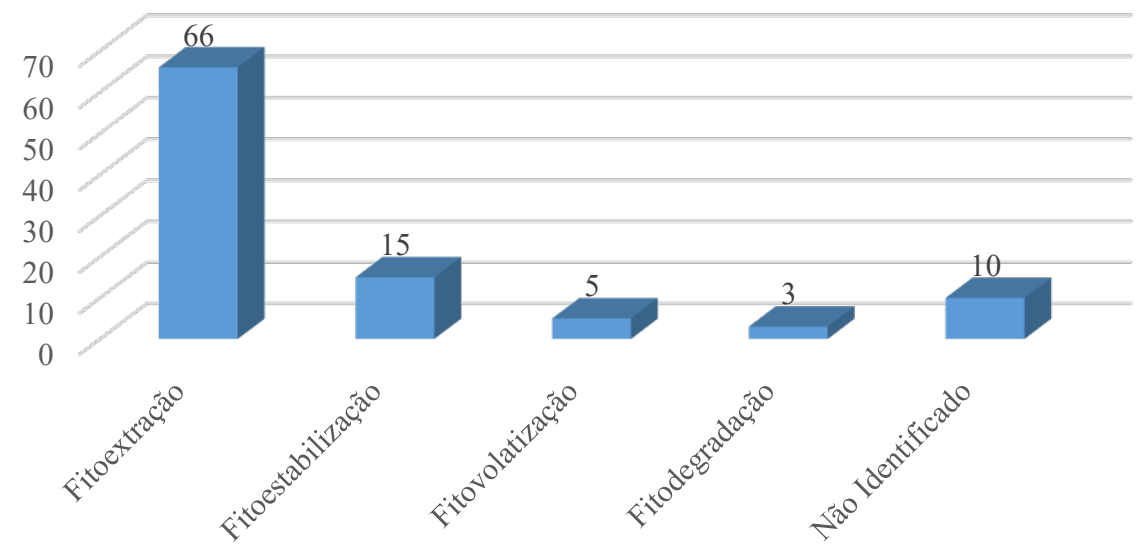

Fonte: Elaborada pelos autores deste artigo (2018)

A fitoextração é uma tecnologia emergente para despoluição de solos contaminados por metais pesados e a mais utilizada atualmente (SILVA, 2012), colaborando com os resultados encontrados neste trabalho.

\section{Considerações Finais}

A fitorremediação é uma técnica promissora para remediar solos contaminados por metais pesados, por ser considerada ambientalmente favorável e de baixo custo. A China é o país que tem o maior número de depósito de patentes, sendo que suas principais universidades têm investido no desenvolvimento da técnica de fitorremediação, principalmente na fitoextração. As patentes sobre fitorremediação são importantes para o desenvolvimento de novos métodos de biorremediação de ambientes impactados por metais pesados, como rejeitos da atividade de mineração. Nesse contexto, evidencia-se que o processo de fitoextração representa o cenário mais estratégico para o investimento em tecnologia de fitorremediação.

\section{Referências}

CAMPOS, M. L.; PIERANGELI, M. A. P.; GUILHERME, L. R. G.; MARQUES, J. J.; CURI, N. Baseline concentration of heavy metals in Brazilian Latosols. Communications in Soil Science and Plant Analysis, [S.1.], v. 34, n. 3-4, p. 547-557, 2003. 
EUROPEAN PATENT OFFICE (Espacenet). Portal virtual. 2017. Disponível em: <https:// worldwide.espacenet.com >. Acesso em: $1^{\circ}$ ago. 2017.

INSTITUTO NACIONAL DE PROPRIEDADE INDUSTRIAL (INPI). Portal virtual. 2017. Disponível em: <http://www.inpi.gov.br/>. Acesso em: $1^{\circ}$ ago. 2017.

MAYERHOFF et al. Instituto Nacional de Propriedade Industrial (INPI). Grupo de Trabalho Especial em Biotecnologia. Estudo comparativo dos critérios de patenteabilidade para invenções biotecnológicas em diferentes países. 2007. Disponível em: <http://www.inpi.gov.br/menuservicos/informacao/arquivos/estudo_comparativo_dos_critriosde_petenteabilidadepara_invenes_ biotecnolgicas_em_diferentes_pases1.pdf > . Acesso em: 17 set. 2017.

NASCIMENTO, C.W. A.; XING, B. Phytoextraction: a review on enhanced metal availability and plant accumulation. Scientia Agricola, São Paulo, v. 63, p. 299-311, 2006.

PUBLICO. Mais de 16,1\% do solo chinês está contaminado, reconheceu o Governo. 2014. Disponível em: <https:/www.publico.pt/2014/04/18/mundo/noticia/mais-de-161-do-solo-chines-estacontaminado-reconheceu-o-governo-1632709>. Acesso em: 3 nov. 2018.

RASKIN, I.; ENSLEY, B. Phytoremediation of toxic metals - using plants to clean up the environment. Plant Science, [S.1.], v. 160, p. 1073-1075, 2000.

RIBEIRO, M. A. C. Contaminação do solo por metais pesados. 2013. Disponível em:

<http://recil.grupolusofona.pt/bitstream/handle/10437/4770/TeseFinalMarcosRibeiro27-01-14. pdf?sequence $=1>$. Acesso em: $1^{\circ}$ set. 2017.

SILVA, J. F. Prospecção de plantas fitorremediadoras em solos contaminados por metais pesados. 2012. $91 \mathrm{f}$. Tese (Doutorado em Biotecnologia) - Programa Multi-institucional de PósGraduação em Biotecnologia. Universidade Federal do Amazonas, Manaus, 2012.

SOBRINHO, Amaral et al. Química dos metais pesados no solo. In: MELO, V. F; ALLEONI, L. R. F. (Ed.). Química e mineralogia do solo: aplicações. Viçosa, MG: SBCS, 2009.

ZUCOLOTO, F. G. Propriedade intelectual e aspectos regulatórios em biotecnologia.

Rio de Janeiro: Ipea, 2013. Cap. 5. Disponível em: < http://protec.org.br/uploads/paginas/file/ Propriedade\%20Intelectual\%20e\%20aspectos\%20regulat\%C3\%B3rios\%20em\%20biotecnologia. pdf $>$. Acesso em: 17 set. 2017.

\section{Sobre os autores}

\section{Sidnei Cerqueira dos Santos}

E-mail: sidnei.cerqueira@unifesspa.edu.br

Doutor em Biotecnologia, pela Universidade Estadual do Ceará (UECE); mestre em Biotecnologia, pela Universidade Estadual de Feira de Santana (UEFS); especialista em Microbiologia, pela Universidade Federal da Bahia (UFBA); e graduado em Ciências Biológicas, pela Universidade Católica do Salvador.

Endereço profissional: Avenida dos Ipês, s/n, Cidade Jardim - Marabá, PA. CEP: 68500-000. 


\section{Marissol Leite}

E-mail: marissoslds@gmail.com

Graduanda em Ciências Biológicas, pela Faculdade de Biologia, Instituto de Estudos em Saúde e Biológicas, Universidade Federal do Sul e Sudeste do Pará PARA na Universidade Federal do Sul e Sudeste do Pará (UNIFESSPA).

Endereço profissional: Avenida dos Ipês, s/n, Cidade Jardim - Marabá, PA. CEP: 68500-000.

\section{Elenara Pereira Ventura Guajajara}

E-mail: elenaraguajajara@unifesspa.edu.br

Graduanda em Ciências Biológicas na Universidade Federal do Sul e Sudeste do Pará (UNIFESSPA); e formada no Curso Técnica em Agroecologia Integrado ao Ensino Médio no Instituto Federal do Pará - Campus Rural de Marabá (CRMB).

Endereço profissional: Avenida dos Ipês, s/n, Cidade Jardim - Marabá, PA. CEP: 68500-000.

\section{Sanderson Silva}

E-mail: sanderson.silva1460@gmail.com

Graduando em Ciências Biológicas, pela Faculdade de Biologia, Instituto de Estudos em Saúde e Biológicas, Universidade Federal do Sul e Sudeste do Pará.

Endereço profissional: Avenida dos Ipês, s/n, Cidade Jardim - Marabá, PA. CEP: 68500-000

\section{Wesley Lima da Silva}

E-mail:weslley_ls@outlook.com,

Graduando em Ciências Biológicas, pela Faculdade de Biologia, Instituto de Estudos em Saúde e Biológicas, Universidade Federal do Sul e Sudeste do Pará.

Endereço profissional: Avenida dos Ipês, s/n, Cidade Jardim - Marabá, PA. CEP: 68500-000 